\title{
A NEW LOOK AT SPITZER PRIMARY TRANSIT OBSERVATIONS OF THE EXOPLANET HD 189733b
}

\author{
G. Morello ${ }^{1,4}$, I. P. Waldmann ${ }^{1}$, G. Tinetti ${ }^{1}$, G. Peres ${ }^{2}$, G. Micela ${ }^{3}$, and I. D. Howarth ${ }^{1}$ \\ ${ }^{1}$ Department of Physics and Astronomy, University College London, Gower Street, WC1E6BT, UK; giuseppe.morello.11@ucl.ac.uk \\ 2 Dipartimento di Fisica, Università degli Studi di Palermo, via Archirafi I-90123, Italy. \\ ${ }^{3}$ Dipartimento di Fisica e Chimica (previously Dipartimento di Fisica), Specola Universitaria, \\ Università degli Studi di Palermo, Piazza del Parlamento 1 I-90123, Italy \\ ${ }^{4}$ INAF-Osservatorio Astronomico di Palermo, Piazza del Parlamento 1 I-90134, Italy \\ Received 2013 December 4; accepted 2014 March 11; published 2014 April 10
}

\begin{abstract}
Blind source separation techniques are used to reanalyze two exoplanetary transit light curves of the exoplanet HD 189733b recorded with the IR camera IRAC on board the Spitzer Space Telescope at $3.6 \mu \mathrm{m}$ during the "cold" era. These observations, together with observations at other IR wavelengths, are crucial to characterize the atmosphere of the planet HD 189733b. Previous analyses of the same data sets reported discrepant results, hence the necessity of the reanalyses. The method we used here is based on the Independent Component Analysis (ICA) statistical technique, which ensures a high degree of objectivity. The use of ICA to detrend single photometric observations in a self-consistent way is novel in the literature. The advantage of our reanalyses over previous work is that we do not have to make any assumptions on the structure of the unknown instrumental systematics. Such "admission of ignorance" may result in larger error bars than reported in the literature, up to a factor 1.6. This is a worthwhile tradeoff for much higher objectivity, necessary for trustworthy claims. Our main results are (1) improved and robust values of orbital and stellar parameters, (2) new measurements of the transit depths at $3.6 \mu \mathrm{m}$, (3) consistency between the parameters estimated from the two observations, (4) repeatability of the measurement within the photometric level of $\sim 2 \times 10^{-4}$ in the IR, and (5) no evidence of stellar variability at the same photometric level within one year.
\end{abstract}

Key words: methods: data analysis - planets and satellites: atmospheres - planets and satellites: individual (HD 189733b) - techniques: photometric

Online-only material: color figures

\section{INTRODUCTION}

Observations of exoplanetary transits are a powerful tool to investigate the nature of planets around other stars. Transits are revealed through periodic drops in the apparent stellar brightness, due to the interposition of a planet between the star and the observer. The shape of an exoplanetary transit light curve depends on the geometry of the star-planet-observer system and the spatial distribution of the stellar emission at the wavelength at which observations are taken (Mandel \& Agol 2002). By solving the inverse problem, it is possible to characterize fully the planet's orbit (period, $P$; semimajor axis, $a$; inclination, $i$; eccentricity, $e$; and argument of periastron, $\omega$ ), and to measure its radius, $r_{p}$ (Seager \& Mallén-Ornelas 2003; Kipping 2008; Mandel \& Agol 2002). Knowledge of the inclination enables determination of the mass of the planet, $m_{p}$, if $m_{p} \sin i$ is known from radial-velocity measurements.

Multiwavelength transit observations can be used to characterize the atmospheres of exoplanets through differences in the transit depths, typically at the level of one part in $\sim 10^{4}$ in stellar flux for giant planets (Brown 2001; Seager \& Sasselov 2000; Tinetti et al. 2007a). For this purpose, the diagnostic parameter is the wavelength-dependent factor $p=r_{p} / R_{s}$, i.e., the ratio between the planetary and the stellar radii (or its square, related to the transit depth).

The exoplanet HD 189733b is one of the most extensively studied hot Jupiters: the brightness of its star allows spectroscopic characterization of the planet's atmosphere.

The $3.6 \mu \mathrm{m}$ transit depth for the exoplanet HD 189733b has been debated in the literature. Different analyses of the same data set, including two simultaneous Spitzer/IRAC observations at $3.6 \mu \mathrm{m}$ and $5.8 \mu \mathrm{m}$, have been used to infer the presence of water vapor in the atmosphere of HD 189733b (Beaulieu et al. 2008; Tinetti et al. 2007b), or to reject this hypothesis (Désert et al. 2009). Another analysis of this data set is reported by Ehrenreich et al. (2007), but we do not comment further their results, as they were not conclusive, because of the very large error bars. Désert et al. (2011) reported the analysis of a second Spitzer/IRAC data set at $3.6 \mu \mathrm{m}$ using the same techniques. Their new estimates of the planet's parameters were significantly different from those reported previously by the same authors (Désert et al. 2009); the discrepancies were attributed by the authors to variations in the star.

Although stellar activity may significantly affect estimates of exoplanetary parameters from transit light curves (Ballerini et al. 2012; Berta et al. 2011), the method used to retrieve the signal of the planet also plays a critical role. The analyses mentioned above were all based on parametric corrections of the instrumental systematics, and are thus, to some degree, subjective. Recently, non-parametric methods have been proposed to decorrelate the transit signals from astrophysical and instrumental noise, and ensure a higher degree of objectivity. Waldmann (2012) and Waldmann et al. (2013) suggested algorithms based on Independent Component Analysis (ICA) to extract information of an exoplanetary atmosphere from Hubble/NICMOS and Spitzer/IRS spectrophotometric data sets.

In this paper, we adopt a similar approach to detrend the transit signal from photometric observations by using pointspread functions (PSFs) covering multiple pixels on the detector. We apply this technique to re-analyze the two observations of primary transits of HD 189733b recorded with Spitzer/IRAC at $3.6 \mu \mathrm{m}$ (channel 1 of IRAC) in the "cold Spitzer" era. We present 
a series of tests to assess the robustness of the method and the error bars of the parameters estimated. Critically, by comparing the results obtained for the two measurements, we discuss the level of repeatability of transit measurements in the IR, limited by the absolute photometric accuracy of the instrument and possible stellar activity effects. We discuss the reliability of our results for orbital and stellar parameters in light of previous multiple $8 \mu \mathrm{m}$ observations (Agol et al. 2010).

\section{DATA ANALYSIS}

\subsection{Observations}

The two Spitzer observations of HD 189733b discussed here were performed on 2006 October 31 (ID 30590) and 2007 November 25 (ID 40732).

The first observation consists of 1936 exposures using IRAC's stellar mode (full-array), taken over $4.5 \mathrm{hr}: 1.8 \mathrm{hr}$ on the primary transit of the planet, $1.6 \mathrm{hr}$ before, and $1.1 \mathrm{hr}$ after transit. The reset time is $8.4 \mathrm{~s}$. During the observation, the centroid of the star HD 189733 was stable to within one pixel.

The second observation was of 1920 exposures using IRAC's sub-array mode, over $4.5 \mathrm{hr} ; 1.8 \mathrm{hr}$ were spent on the primary transit of the planet, $1.7 \mathrm{hr}$ before, and $1 \mathrm{hr}$ after transit. The interval between consecutive exposures is $8.4 \mathrm{~s}$. Each exposure consists of 64 reads at high speed cadence of $0.1 \mathrm{~s}$. Only for the ObsID 40732, we replaced the 64 reads of each exposure with their mean values, in order to have a manageable number of data points, to reduce the random scatter, and to have the same sampling of the ObsID 30590. During the observation, the centroid of the star HD 189733 was again stable to within one pixel.

\subsection{Independent Component Analysis in the Context of Exoplanetary Transits Light Curves}

ICA consists of a transformation from a set of recorded signals to an equivalent set of maximally independent components. The underlying assumptions are that:

1. each recorded signal is a linear combination of the same source signals;

2. the source signals are mutually independent.

We can express this model as

$$
\begin{gathered}
x_{1}=a_{1,1} s_{1}+a_{1,2} s_{2}+\ldots+a_{1, n} s_{n} \\
x_{2}=a_{2,1} s_{1}+a_{2,2} s_{2}+\ldots+a_{2, n} s_{n} \\
\vdots \\
x_{n}=a_{n, 1} s_{1}+a_{n, 2} s_{2}+\ldots+a_{n, n} s_{n}
\end{gathered}
$$

where $x_{i}, i=1 \ldots n$, are the recorded signals, $s_{j}, j=1 \ldots n$, are the source signals, and $a_{i, j}$ are numerical coefficients. Equation (1) can be written in matrix form as

$$
\mathbf{x}=\mathbf{A s}
$$

where $\mathbf{x}$ is the column vector containing the recorded signals, $\mathbf{s}$ is the column vector containing the source signals, and $\mathbf{A}$ is the matrix of the coefficients, the so-called mixing matrix.

The aim of ICA is the "blind" separation of the source signals from the observations, i.e., without any additional information (except the assumed mutual independence of the source signals). In other words, the ICA algorithms search for the matrix $\mathbf{W}$ that transforms the recorded signals such that the mutual statistical independence is maximized:

$$
\mathbf{W x}=\mathbf{W A s}
$$

If the assumptions are valid, then $\mathbf{W A}=\mathbf{D}$, where $\mathbf{D}$ is a diagonal matrix, so that

$$
\mathbf{W x}=\mathbf{D s}
$$

The diagonal matrix $\mathbf{D}$ means that the extracted signals can be rescaled without changing the mutual independence.

To maximize the said independence, several approaches and implementations have been proposed (Hyvärinen et al. 2001; Tichavský et al. 2008). We used the MULTICOMBI algorithm (Tichavský et al. 2008), which optimally mixes EFICA and WASOBI, based on maximizing the non-Gaussianity of the extracted signals (Koldovský et al. 2006) and their temporal decorrelations (Yeredor 2000), respectively.

In this work, the observed signals are light curves of a star, recorded for a time interval that includes an exoplanetary transit event. These light curves contain at least three independent contributing signals:

1. the astrophysical signal,

2. the signal of instrumental systematics, and

3. stochastic noise.

It is possible, in principle, to decompose further the astrophysical and instrumental systematics signals. The former is the sum of the transit signal, the astrophysical background, possible stellar activity signals, etc., the latter is the sum of different effects from different parts of the instrumentation. All these signals are expected to be independent from each other as they have different origins. By contrast, their linear combinations (i.e., the observed light curves) are clearly not mutually independent. It is worth stressing that to disentangle effectively all these signals, we need, at least, the number of available light curves to be equal to the number of signals. Therefore, we need light curves recorded with the same instrument (since light curves recorded with different instruments have different systematics plus the astrophysical signals, so that the number of source signals is greater than the number of light curves). In principle, using light curves recorded at different times with the same instruments should not work; although the systematics have the same origins, the relevant signals are not necessarily in phase, and so may differ by more than a simple scaling factor. Additionally, further differences might be present due to stellar variability. However, the transit signal, being common to all the light curves, is potentially detrendable. A successful extraction of a transit signal from a time series spanning several exoplanetary transit events, conveniently split into sub-light curves, is described in Waldmann (2012).

The advantage of spectroscopic observations over photometry is the provision of simultaneous light curves at different wavelengths with largely common instrumental systematics. The transit signals at each wavelength can be obtained by subtracting proper systematics models from the light curves (an accurate direct extraction of the transit is impossible due to the limb darkening effect). By using this technique, Waldmann et al. (2013) extracted an infrared transmission spectrum of HD $189733 \mathrm{~b}$ between $1.51 \mu \mathrm{m}$ and $2.43 \mu \mathrm{m}$ from a Hubble/ NICMOS data set. 


\subsection{ICA Using Pixel-light Curves}

The main novelty of the algorithms we use here is their ability to detrend the transit signal from a single photometric observation of just one primary transit. This is possible because, even if stars can be approximated by point sources, the instrument is purposely de-focused to spread the PSF over several detector pixels, and the position of the target star on the detectors is stable to within one pixel. During an observation, there are several pixels detecting the same astrophysical signals at any time, but with different scaling factors, depending on their received flux, their quantum efficiency, and the instrument PSF.

We performed an ICA decomposition over several pixellight curves, i.e., the time series from individual pixels, in order to extract the transit signal and other independent signal components (stellar or instrumental in nature).

Once a set of independent components has been obtained from a selected set of pixel-light curves, different approaches to obtain the transit signal can be considered.

Method 1: direct identification of the transit component. In principle, if one of the independent components extracted has the morphology of the transit signal, we assume that one to be the transit signal, multiplied by an undetermined scaling factor. We renormalize the signal by the mean value calculated on the out-of-transit part, so that the out-of-transit level is unity.

Method 1 is not applicable to the extraction of accurate transit signals from spectroscopically resolved observations of a primary transit at different wavelengths because of the wavelength dependence of stellar limb darkening. This is not a problem in our case because all the pixels record the same wavelengths.

Method 2: non-transit-components subtraction. Another approach to estimating the transit signal is to remove all the other effects from an observed light curve, i.e., by subtracting all the components other than the transit one, properly scaled. The scaling factors can be determined by fitting a linear combination of the components, plus a constant term, to the out-of-transit part of the light curve. ${ }^{5}$ The coefficients of the linear combination and the constant are the free parameters to fit.

Instead of fitting the non-transit-components on the pixellight curves and then subtracting, we performed these processes on the spatially integrated light curves, obtained by summing all the individual pixel-light curves. The integrated light curves are much less noisy than the individual pixel curves.

\subsection{Transit Light Curve Fitting and Error Bars}

After the extractions of the detrended and normalized transit time series, we modeled them using the Mandel \& Agol (2002) analytical formulae. We can compute the observed flux as a function $F(p, z)$, where $p=r_{p} / R_{s}$ is the ratio between the planetary and the stellar radii, and $z=d / R_{s}$ is the distance between the centers of their disks projected onto the sky divided by the stellar radius. The relative distance $z$ is a function of time, determined by the orbital parameters.

We assumed the orbital period $P$, zero eccentricity, and a quadratic limb darkening model (Howarth 2011). The values of the fixed parameters are reported in Table 1.

\footnotetext{
5 The out-of-transit limits do not have to be known exactly. They can be chosen in a way to ensure that part of the transit is not included while fitting, relying on parameters reported in previous papers and on the light curves themselves. The results should not be affected by this choice, but it is worth checking this point.
}

Table 1

Values of the Parameters Fixed While Generating the Transit Models

\begin{tabular}{lc}
\hline \hline$P$ & 2.218573 days \\
$e$ & 0 \\
$\gamma_{1}$ & $7.82118 \times 10^{-2}$ \\
$\gamma_{2}$ & $2.00656 \times 10^{-1}$ \\
\hline
\end{tabular}

Notes. The limb darkening coefficients, $\gamma_{1}$ and $\gamma_{2}$, were computed for a star with effective temperature $T_{\text {eff }}=$ $5000 \mathrm{~K}$, gravity $\log g=4.5$, mixing-length parameter $l / h=1.25$, and solar abundances.

We first determined the centers of the transit ephemeris by fitting some symmetric models with all other parameters fixed. Recent papers (Collier Cameron et al. 2010; Triaud et al. 2010) report a small but non-zero eccentricity $\left(e \simeq 4 \times 10^{-3}\right.$ ), but we verified that this would affect our estimates of the other parameters by a negligible fraction of their error bars.

We then performed a fit with three free parameters:

1. the ratio of planetary to stellar radii, $p=r_{p} / R_{s}$,

2. the orbital semimajor axis (in units of the stellar radius), $a_{0}=a / R_{s}$, and

3. the orbital inclination, $i$.

We chose these as free parameters, because

1. there is a large range of values published in the literature,

2. they largely determine the shape of the transit signal, and

3 . they do not show strong cross-correlations.

For completeness, and for comparisons with the literature, in the final results we also report the transit depth $p^{2}$, the impact parameter $b$, and the duration of the transit $T$, where

$$
\begin{aligned}
& b=a_{0} \cos i \\
& T=\frac{P \sqrt{1-b^{2}}}{\pi a_{0}} .
\end{aligned}
$$

We used a Nelder-Mead optimization algorithm (Lagarias et al. 1998) to obtain first estimates of the parameters of a model. To confirm/improve these estimates and to determine error bars, we ran an Adaptive Metropolis algorithm with delayed rejection (Haario et al. 2006) for 20,000 iterations, starting from the optimal values initially determined, in order to sample the probability distributions of the fitted parameters. The updated best estimates and error bars of the parameters are the means and the standard deviations of the sampled distributions (approximately Gaussian), respectively. No burn-in is required because of the optimal starting points of the chains.

The variance of the likelihood function is initialized as the variance of the residuals obtained for the first model and then sampled together with the other free parameters $\left(\sigma_{0}^{2}\right)$. In this way, we take into account both white and the autocorrelated noise present in the detrended time series, but we ignore possible systematic errors due to the preliminary ICA deconvolution. The ICA errors can be represented as an additional uncertainty, $\sigma_{\text {ICA }}$, on each point in the time series. The likelihood's variance, $\sigma_{\text {like }}^{2}$, becomes

$$
\sigma_{\text {like }}^{2}=\sigma_{0}^{2}+\sigma_{\text {ICA }}^{2}
$$

We tested that resampling the parameters' chains with $\sigma_{\text {like }}^{2}$ does not affect their best values, while the total error bars of the single 

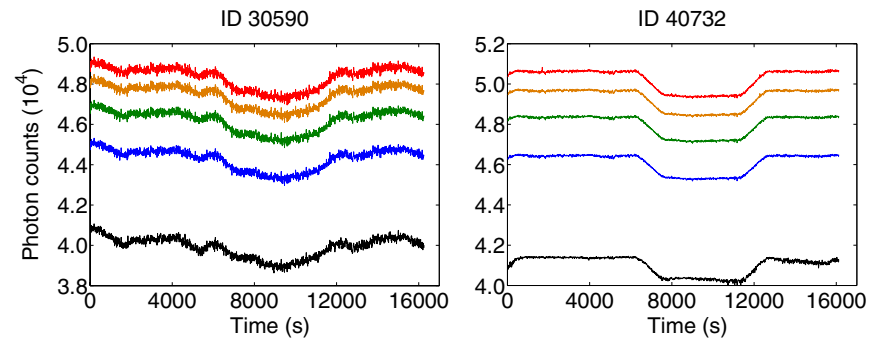

Figure 1. Raw integral light curves from several squared arrays of pixels: black $3 \times 3$, blue $5 \times 5$, green $7 \times 7$, orange $9 \times 9$, and red $11 \times 11$ (in order of increasing counts).

(A color version of this figure is available in the online journal.)

parameters, $\sigma_{\text {par }}$, increase with respect to the previous estimates (without the ICA errors), $\sigma_{\mathrm{par}, 0}$, as

$$
\sigma_{\mathrm{par}}=\sigma_{\mathrm{par}, 0} \frac{\sigma_{\text {like }}}{\sigma_{0}}=\sigma_{\mathrm{par}, 0} \sqrt{\frac{\sigma_{0}^{2}+\sigma_{\mathrm{ICA}}^{2}}{\sigma_{0}^{2}}} .
$$

A measure of the uncertainties on the independent components extracted by ICA is given by the interference-to-signal ratio matrix, ISR, i.e., an $n \times n$ matrix, where $n$ is the number of signals. The $\mathrm{ISR}_{i j}$ element estimates the relative remaining presence of the $j^{\text {th }}$ component in the $i^{\text {th }}$ one. Then,

$$
\mathrm{ISR}_{i}=\sum_{j=1, j \neq i}^{n} \mathrm{ISR}_{i j}
$$

estimates the relative remaining presence of all the other components in the $i^{\text {th }}$ one.

If the $i^{\text {th }}$ component represents the transit signal, and if we estimate the transit signal through method 1, we can identify

$$
\sigma_{\mathrm{ICA}}^{2}=f^{2} \mathrm{ISR}_{i}
$$

with $f$ the scaling factor used.

If the $i^{\text {th }}$ component represents the transit signal, but we estimate it through method $2, \sigma_{\text {ICA }}$ has to contain a weighted sum of the ISRs of the non-transit components removed, plus the discrepancies of the fit to the out-of-transit phases:

$$
\sigma_{\mathrm{ICA}}^{2}=f^{2}\left(\sum_{j=1}^{m} o_{j}^{2} \mathrm{ISR}_{j}+\sigma_{\mathrm{ntc}-\mathrm{fit}}^{2}\right),
$$

$o_{j}$ being the coefficients of the non-transit components, $m$ the number of components considered, $\sigma_{\text {ntc-fit }}$ the standard deviation of the residuals from the reference light curve (out of the transit), and $f$ the normalizing factor for the model-subtracted light curve.

The MULTICOMBI code produces two ISR matrices, $\mathrm{ISR}^{\mathrm{EF}}$, associated with the algorithm EFICA, and the ISR ${ }^{\mathrm{WA}}$, associated with the algorithm WASOBI. We estimated the global ISR as their average:

$$
\mathrm{ISR}=\frac{\mathrm{ISR}^{\mathrm{EF}}+\mathrm{ISR}^{\mathrm{WA}}}{2} .
$$

This is a conservative estimate, given that, according to Tichavský et al. (2008), the MULTICOMBI ISR slightly outperforms the best of ISR ${ }^{\mathrm{EF}}$ and ISR ${ }^{\mathrm{WA}}$ (then it could be smaller), but these estimates are entirely reliable only under certain assumptions on the signals which may not be satisfied in these cases. Here we take them as worst-case estimates.

\subsection{Application to Observations}

Here, we describe the main steps of the analyses performed on each of the two observations (ID 30590 and ID 40732), which include some tests of robustness. We now discuss only results obtained with method 2, as they are much more stable; results obtained with method 1 are reported in Appendix C, along with a critical comparison of the two methods.

\subsubsection{Choice of the Pixels}

The first step in the analysis is the choice of the pixel-light curves to analyze. This is determined by

1. the instrument point response function (PRF), i.e., the measured intensity profile of the star on the detector ${ }^{6}$,

2. the noise level of the detector, and

3 . the effective number of significant components to disentangle.

The number of significant components is not known a priori. The ICA code extracts a number of components equal to the number of light curves that it receives as input. Apart from the collective behavior common to all the pixel-light curves, each pixel introduces an individual signature. Only if the individual signatures are negligible compared to the collective behavior are we able to select enough light curves to disentangle the significant components. The PRF and the noise level of the detector limit the number of pixels containing potentially useful astrophysical information.

In practice, we considered several arrays of pixels with the stellar centroid at their centers, having dimensions $3 \times 3,5 \times 5$, $7 \times 7,9 \times 9$, and $11 \times 11$ pixels. Figure 1 shows the "integral light curves," obtained by summing the contributions from the various pixels. We looked for outliers in the time series, i.e., points discrepant more than $5 \sigma$ from a first transit-light curve model (fitted on the original data), and we replaced those outliers with the averages of the points immediately before and after. We found only one outlier in ObsID 30590, and nine in ID 40732. Although the observed light curves are two primary transits of the same exoplanet, observed at the same wavelength through the same instrument, they appear very different, mainly because of the different observing strategies. In particular, ObsID 40732 seems to be much less affected by systematics, and less noisy. The integral light curves from the various arrays of pixels look very similar in shape, but have different absolute intensities, as expected. The mean intensities of the integral $3 \times 3,5 \times 5,7 \times 7$, and $9 \times 9$ light curves are, respectively, $\sim 83 \%, \sim 92 \%, \sim 96 \%$, and $\sim 98 \%$ of the mean intensity of the integral $11 \times 11$ light curve. We are not interested in absolute photometry, but only in relative variations of the intensity, therefore it is not important whether the PRF is totally contained in the square used for the analysis or not, provided it contains enough information to detrend the transit signal. Larger arrays include pixels which add noise with little or no astrophysical information. We concluded that the $3 \times 3$ or the $5 \times 5$ arrays were the optimal choices. However, we tested all the pixel arrays to assess the robustness of the results.

We binned the transit time series by replacing groups of nine consecutive points with their mean values in order to reduce the computational time required to sample the parameters' distributions in the Mandel \& Agol (2002) model (see Section 2.4).

\footnotetext{
6 Note that the PRF is, in principle, slightly different from the PSF: the PSF is the intensity profile incident on the detector, while the PRF is the measured intensity profile (including the detector response).
} 


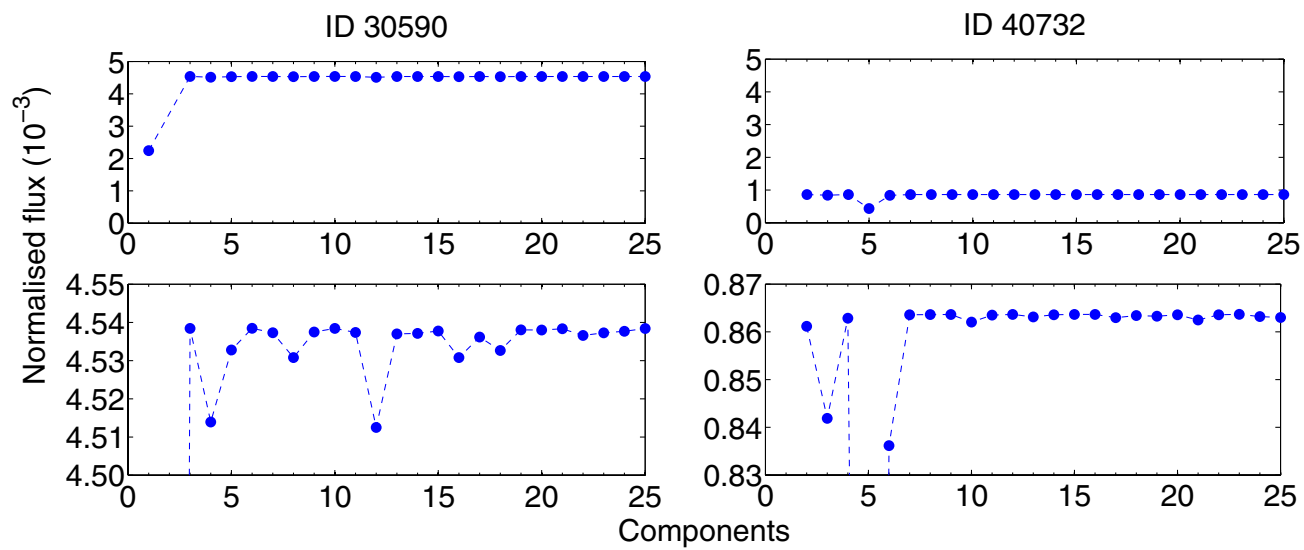

Figure 2. Top: standard deviations of the residuals of the single-component fits, normalized to out-of-transit level; $5 \times 5$ array. Bottom: the same, zooming on the topmost part of the curve.

(A color version of this figure is available in the online journal.)

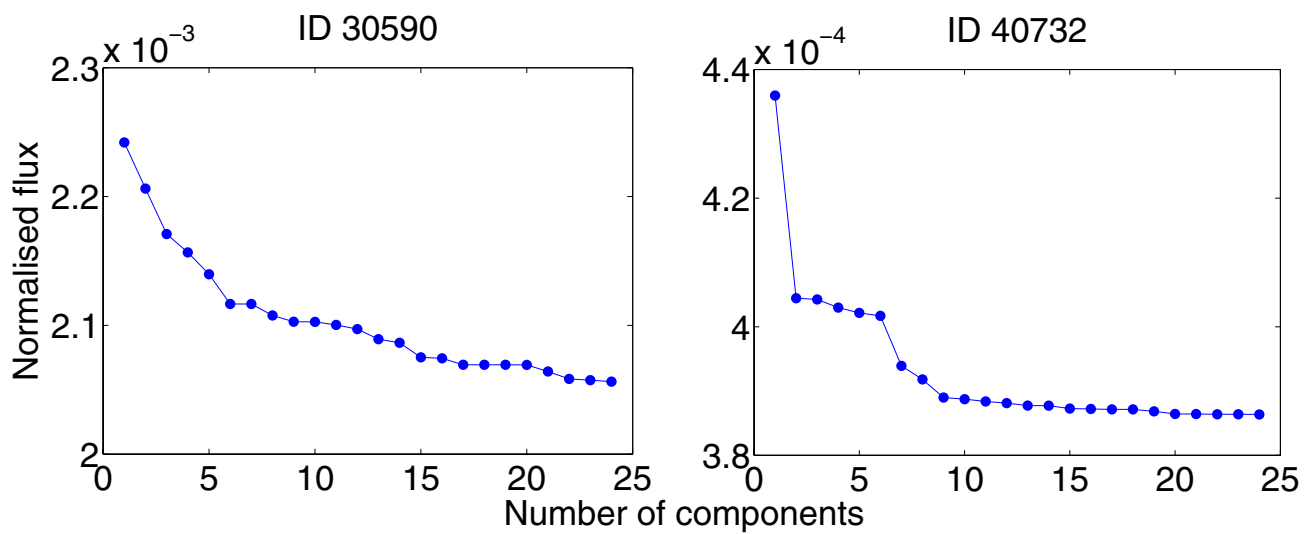

Figure 3. Standard deviations of the residuals of the fits with multiple components, normalized to out-of-transit level, $5 \times 5$ array.

(A color version of this figure is available in the online journal.)

We checked that in select cases this approach does not affect the parameter estimates.

The best values of $p, a_{0}$, and $i$ are stable, within the error bars, with respect to the choice of the set of pixel-light curves used to detrend the signals. The discrepancies between the extracted signals and the relative fits are the biggest for the $3 \times 3$ array; for larger arrays they are smaller, and are either all at the same level (ID 30590) or slightly decrease with the size of the array (ID 40732). Our interpretation of this is that the $5 \times 5$ and larger arrays contain the same amount of useful information, while in the $3 \times 3$ array something is missed. The ICA errors confirm this hypothesis, being the smallest for the $7 \times 7$ (ID 30590) and $5 \times 5$ (ID 40732) arrays. Higher values for larger arrays were expected, but do not differ significantly. We conclude that the choice of the array size is not crucial.

\subsubsection{Choice of the Components}

In Section 2.5.1, we corrected the observed light curves by subtracting all the non-transit components (see Section 2.3). Here, we show how to identify the most significant components, and how many should be considered. We generally expect that some components are related to collective behaviors common to all the pixels, and others to individual pixels' signatures and/ or noisy mixtures of the sources. By inspection, a few of the components clearly present time structures, while others have randomly scattered time series.
We report results from the $5 \times 5$ array only, as it is the smallest array containing all the astrophysical and instrumental information.

To evaluate the impact of each component in the out-of-transit data, we found the best fits of the single components (plus additive constants) to that part of the integral light curve, and calculated the means and standard deviations of the residuals. In this way, we established a ranking of importance of the components, based on the minimization of the discrepancies between their fits and the integral light curve, out of transit. We then computed other best fits by using the $n$ most important components, according to that ranking, with $n$ from 1 to 24 . The best coefficients for the components and the additive constants were determined through the Nelder-Mead optimization algorithm.

Figure 2 reports the standard deviations of the residuals of the single-component fits, normalized to the out-of-transit level and Figure 3 reports analogous fits obtained using more components. Note that figures related to different observations are not reported with the same scale because they have very different accuracies. Most systematics are contained in one major component, but the use of multiple components increases the detrending accuracy.

We computed 24 estimates of the transit signal by removing the $n$ most significant non-transit components from the integral light curve. We binned these over nine points, as in Section 2.5.1, and fitted Mandel \& Agol (2002) models to these curves. The 


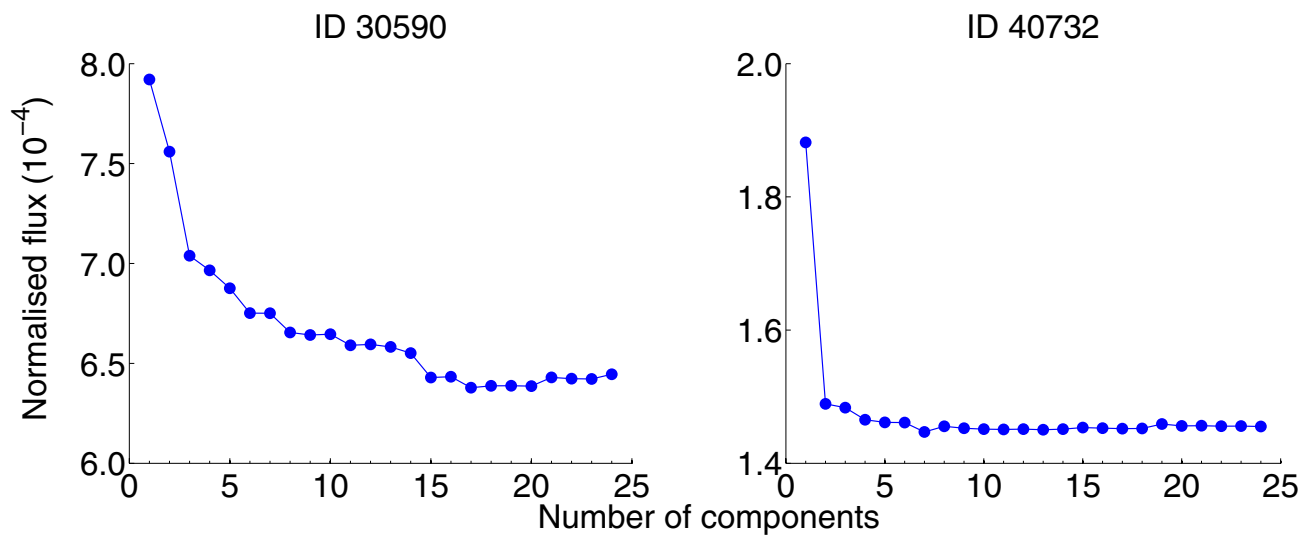

Figure 4. Standard deviations of the residuals between the transit signals estimated using method 2 (with the $n$ most important components, binned by nine points), and the corresponding best model fits.

(A color version of this figure is available in the online journal.)

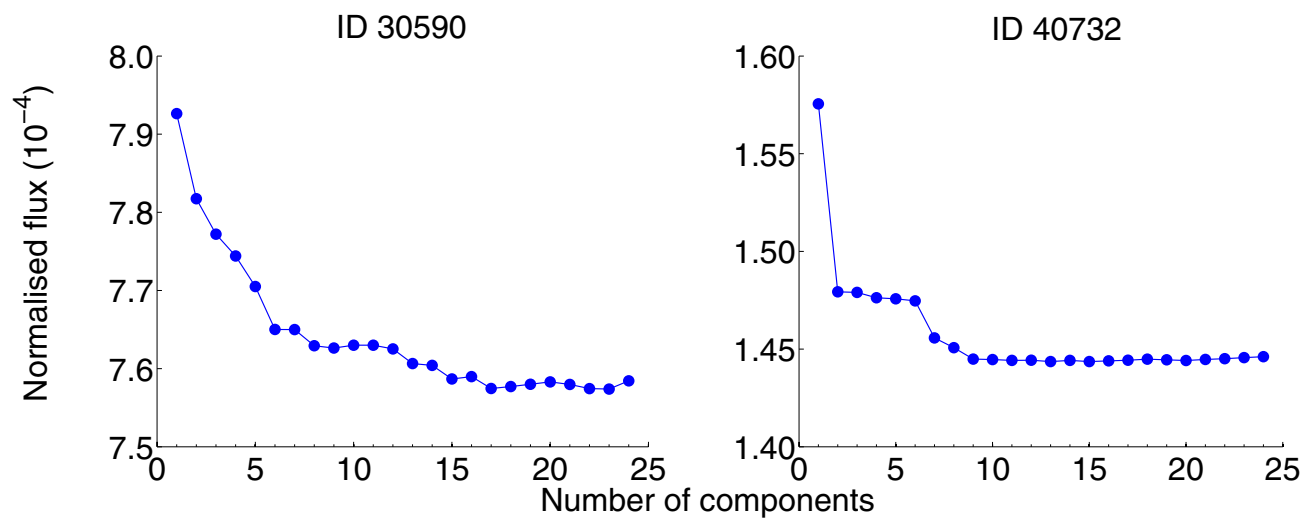

Figure 5. ICA separation errors for the transit signals estimated through method 2 (with the $n$ most important components, binned by nine points).

(A color version of this figure is available in the online journal.)

standard deviations of the residuals between each curve and the corresponding best model of Mandel \& Agol (2002) are reported in Figure 4, and confirm that the use of multiple components for detrending improves the results.

ICA separation errors are plotted in Figure 5, showing the same trends.

Given these tests, we expect to have good estimates of the transit signals by removing the first few most significant components, but some improvements can be made by removing more components, up to a saturation point. The best values of the parameters $p, a_{0}$, and $i$ for each estimated transit signal are shown in Figure 12.

The dispersions in the parameters are fully contained in the intervals previously estimated by using the signals with all non-transit components removed (see Appendix A, Table 6, Column 2), except for values from the transit signal from ObsID 40732 with only one component removed.

\section{RESULTS}

Figure 6 shows the normalized transit signals extracted using the $5 \times 5$ arrays, considering all the independent components, the relative best light curve fits to the binned and detrended data, and the residuals. The standard deviations of the residuals are $\sigma_{0}^{\mathrm{ID} 30590}=6.4 \times 10^{-4}$ and $\sigma_{0}^{\mathrm{ID}} 40732=1.45 \times 10^{-4}$. Note that the signal extracted from ObsID 40732 has a dispersion smaller by a factor $\sim 4.4$.
Table 2

Estimated $p, a_{0}$, and $i$ from the $5 \times 5$ Array of Pixels, Through Method 2, Considering All the Independent Components, without Including the ICA Error (See Sections 2.3 and 2.4)

\begin{tabular}{lccc}
\hline \hline & Starting Value & Mean & Standard Deviation \\
\hline$p$ & & ID 30590 & \\
$a_{0}$ & 0.15471 & 0.15470 & $3.6 \times 10^{-4}$ \\
$i$ & 9.05 & 9.06 & 0.09 \\
\hline & 85.93 & 85.94 & 0.10 \\
\hline$p$ & & ID 40732 & $8 \times 10^{-5}$ \\
$a_{0}$ & 0.15534 & 0.15534 & 0.02 \\
$i$ & 8.92 & 8.92 & 0.02 \\
\hline
\end{tabular}

Figure 7 illustrates the sampled distributions of the parameters $p, a_{0}$, and $i$, from the transit signal extracted by ObsID 40732 (see Section 2.4). Similar distributions, but with larger dispersions, were obtained for the other transit signal. Table 2 reports the starting values, sampled means, and standard deviations of the parameters obtained. Note that the starting values agree very well with the sampled means. The likelihood variances without the ICA contribute, calculated as detailed in Section 2.4, are equal to the variances of the residuals: $\sigma_{0}^{\text {ID } 30590}=(6.5 \pm 0.3) \times$ $10^{-4}$, and $\sigma_{0}^{\mathrm{ID} 40732}=(1.46 \pm 0.07) \times 10^{-4}$. 

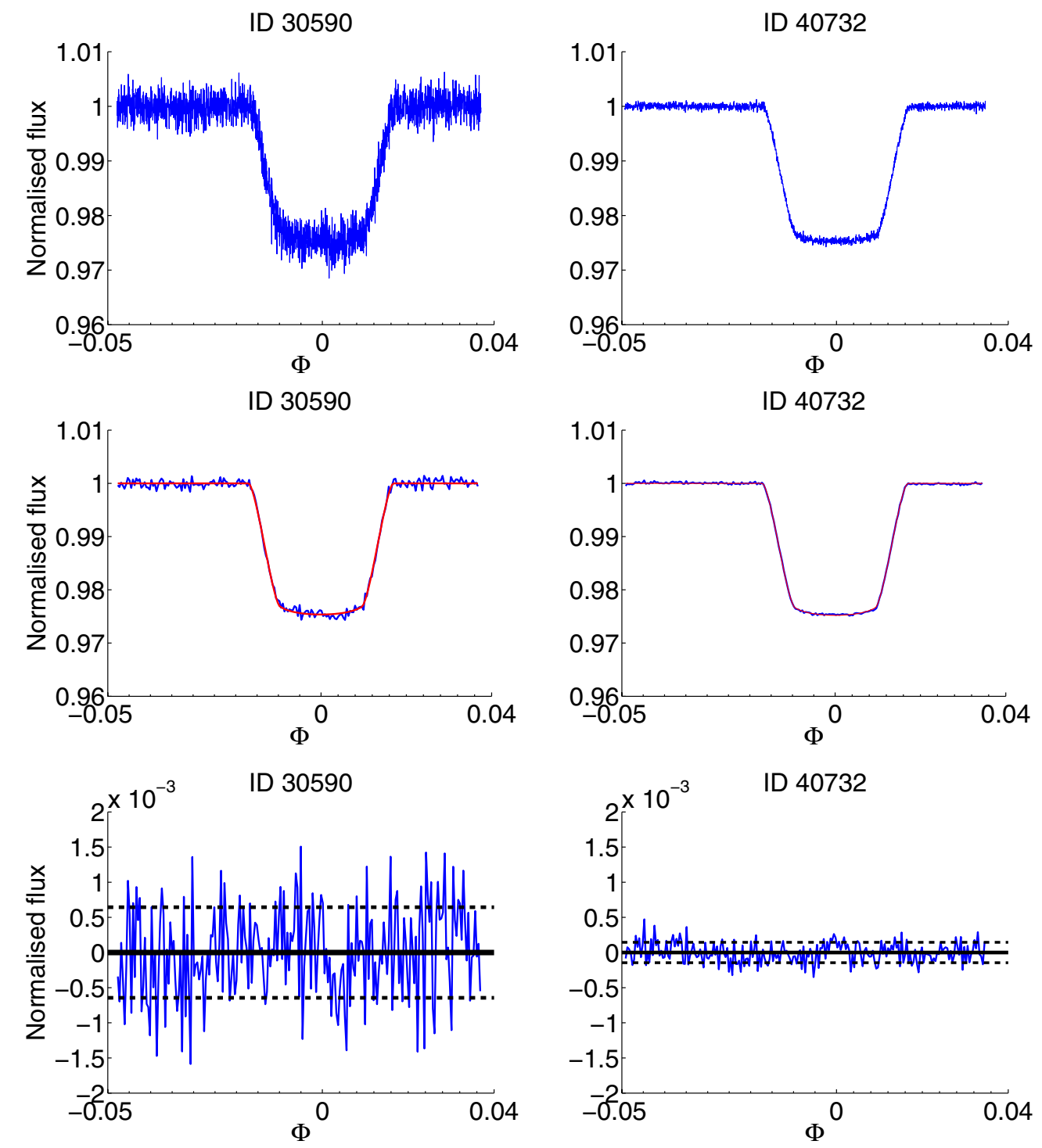

Figure 6. Top panel: transit time series extracted using the $5 \times 5$ array, considering all the independent components (see Section 2.3). Middle panel: (blue) the same series, binned by nine points, (red) relative best model fit. Bottom panel: residuals between the extracted time series and the model. Dashed black lines indicate the standard deviations of the residuals.

(A color version of this figure is available in the online journal.)

Table 3

Adopted Best Estimates of Parameter Values

\begin{tabular}{lcc}
\hline \hline & ID 30590 & ID 40732 \\
\hline$p$ & $0.1547 \pm 0.0005$ & $0.15534 \pm 0.00011$ \\
$a_{0}$ & $9.05 \pm 0.16$ & $8.92 \pm 0.03$ \\
$i$ & $85.93 \pm 0.15$ & $85.78 \pm 0.03$ \\
$p^{2}$ & $0.02394 \pm 0.00017$ & $0.02413 \pm 0.00003$ \\
$b$ & $0.64 \pm 0.03$ & $0.657 \pm 0.005$ \\
$T$ & $5170 \pm 200 \mathrm{~s}$ & $5157 \pm 34 \mathrm{~s}$ \\
\hline
\end{tabular}

Table 3 gives the final results for the parameters $p, a_{0}, i, p^{2}$, $b$, and $T$.

\subsection{Combining Observations}

The parameter estimates determined from ObsID 40732 are much more accurate than those from ID 30590. Assuming that the orbital parameters were the same along the two observations, as expected because of the stability of the planetary orbit, we computed a chain for ID 30590, for $p$ only, with $a_{0}$ and $i$ fixed to the best values estimated from ID 40732. In this way, we can make a direct comparison of $p$ between the two observations, and avoid possible correlations with the other parameters. Even if $a_{0}$ and $i$ were badly determined due to an inaccurate stellar model being assumed (e.g., wrong limb darkening coefficients, star spots, or faculae), they would introduce a systematic error on $p$ that would be equal for both observations. Thus variations of $p$ (or $p^{2}$ ), obtained while keeping all other parameters fixed, are a more objective measurement of the stellar variations. Results are reported in Table 4; note that $\sigma_{0}$ is unchanged. Figure 8 shows the discrepancies between the detrended signal and the model. Including the ICA errors, we found

$$
\begin{gathered}
p=0.1551 \pm 0.0004 \\
p^{2}=0.02405 \pm 0.00014
\end{gathered}
$$



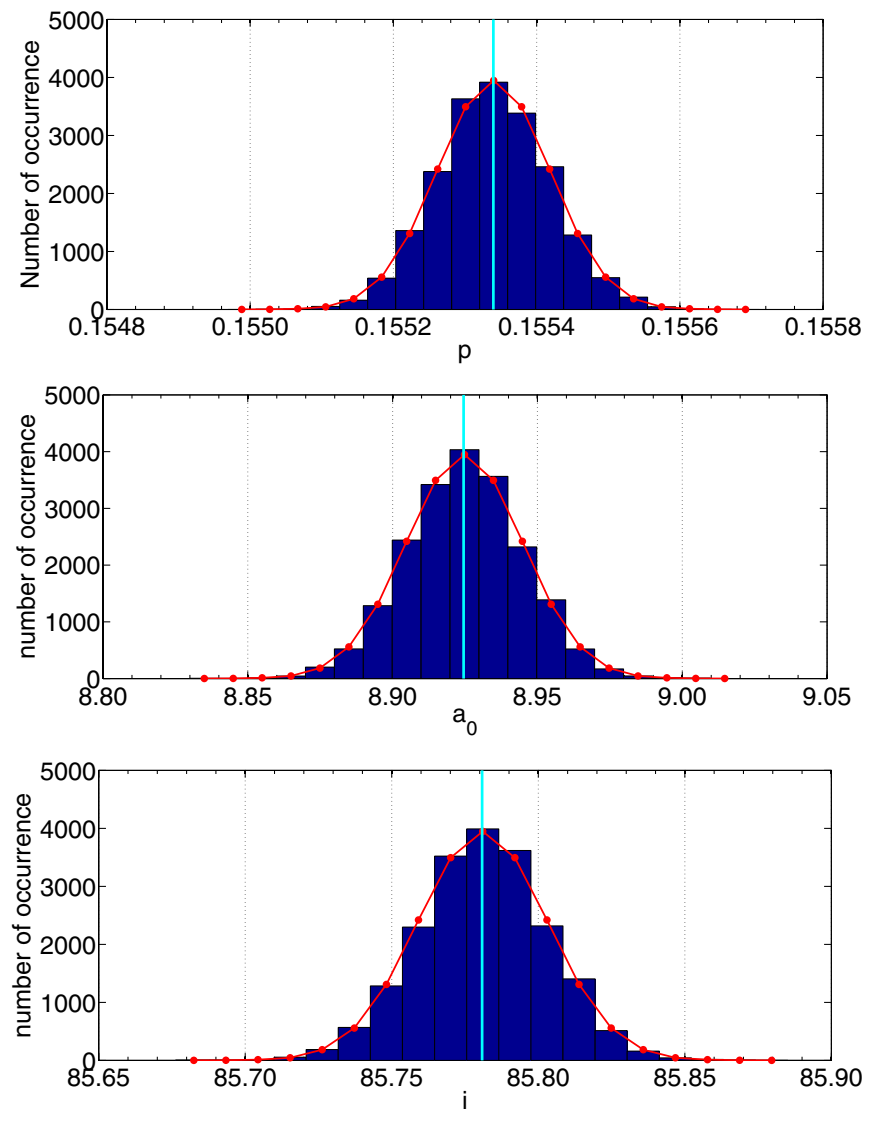

Figure 7. From top to bottom, histograms of the sampled chains for parameters $p, a_{0}$, and $i$, respectively, relative to the time series estimated from the $5 \times 5$ array, considering all the independent components, without including the ICA error (ObsID 40732). The overplotted red curves show Gaussian distributions with the sampled means and variances. The light blue vertical lines indicate the starting values, determined through the Nelder-Mead optimization (see Sections 2.3 and 2.4).

(A color version of this figure is available in the online journal.)

Table 4

Estimated Best Values and Standard Deviations of $p$, from ObsID 30590, with $a_{0}=8.92$ and $i=85.78$, without Including the ICA Error

\begin{tabular}{lc}
\hline \hline$p$ & 0.15507 \\
$\sigma_{p}^{0}$ & $2.7 \times 10^{-4}$ \\
\hline$\sigma_{0}$ & $6.5 \times 10^{-4}$ \\
\hline
\end{tabular}

Note. Discrepancies between the signals and the related best model fits (see Section 2.4).

Figure 9 compares the original estimates for $p$ and $p^{2}$ with those obtained by keeping $a_{0}$ and $i$ fixed. We note that

1. the best value from ID 30590 with $a_{0}$ and $i$ fixed agrees better with the result from ID 40732 and

2. the new estimate from ID 30590 is consistent with the previous one, but with a (slightly) smaller error bar.

\section{DISCUSSION}

\subsection{Comparison of the Two Observations}

The planetary, orbital, and stellar parameters derived separately from the two observations are all consistent within $1 \sigma$. In

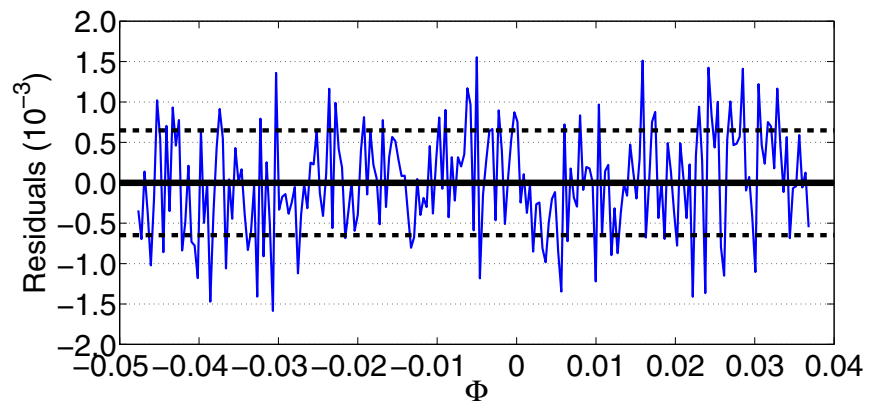

Figure 8. Residuals between the transit signal from ObsID 30590 and the model fit with $a_{0}=8.92$ and $i=85.78$. Black dashed lines indicate the standard deviation, which is consistent with the standard deviation of the residuals between the signal and the model estimated with $a_{0}$ and $i$ as free parameters.

(A color version of this figure is available in the online journal.)

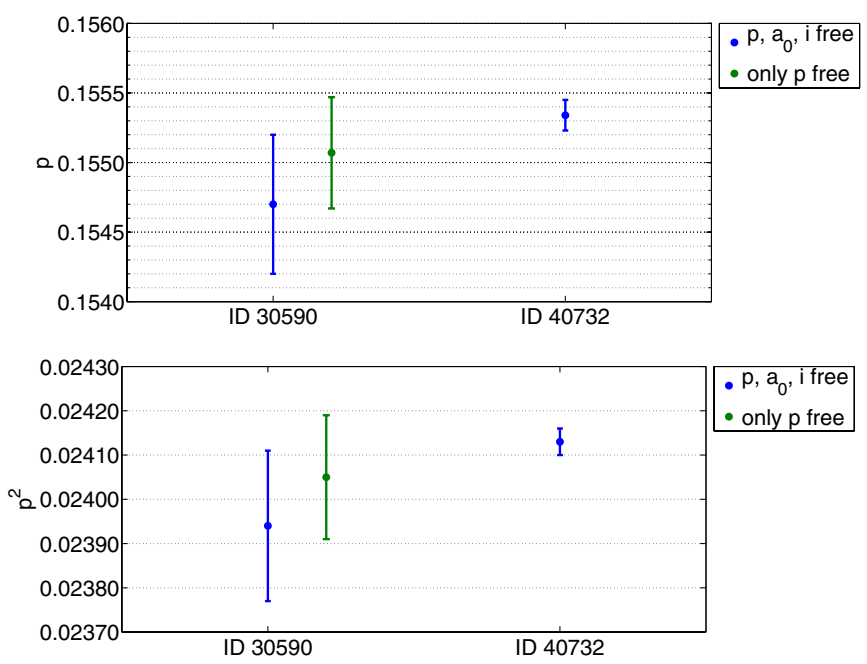

Figure 9. Top: estimates of $p$; with $a_{0}$ and $i$ free (blue); with $a_{0}=8.92$, and $i=85.78$, i.e., the best values found for ObsID 40732 (green). Bottom: the same for $p^{2}$.

(A color version of this figure is available in the online journal.)

particular, the duration of the transit is extremely stable between the two observations. This is not surprising, because its measure is almost insensitive to calibration errors and stellar activity; all other parameters are much more affected by these sources of noise. Furthermore, these other parameters are strongly correlated; e.g., a non-optimal estimate of the impact parameter $b$ will result in an imprecise transit depth $p$, etc. Figure 10 shows the differences between the transit signals extracted for the two observations. The standard deviation of the differences is $\sim 6.8 \times 10^{-4}$, which is comparable with the standard deviation of the discrepancies between the signal from ObsID 30590 and the relative model fit $\left(\sigma_{0}^{\mathrm{ID} 30590}=(6.5 \pm 0.3) \times 10^{-4}\right)$; the discrepancies between the signal from ObsID 40732 and the corresponding model fit are negligible. Hence, there is no evidence of physical variations in the transit signal from one observation to the other one.

The results of Section 3.1 reinforce our claim of nondetectable stellar activity variations.

We conclude that the two observations lead to consistent results, but the second constrains the orbital and stellar parameters much better and allows the estimate of the transit depth for the first one to be refined. 


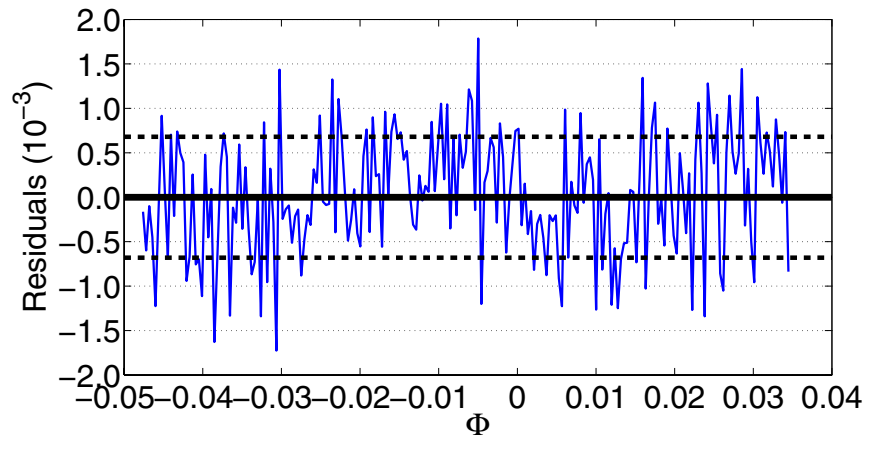

Figure 10. Residuals obtained by subtracting ObsID 40732 to ObsID 30590. Black dashed lines indicate their standard deviation.

(A color version of this figure is available in the online journal.)
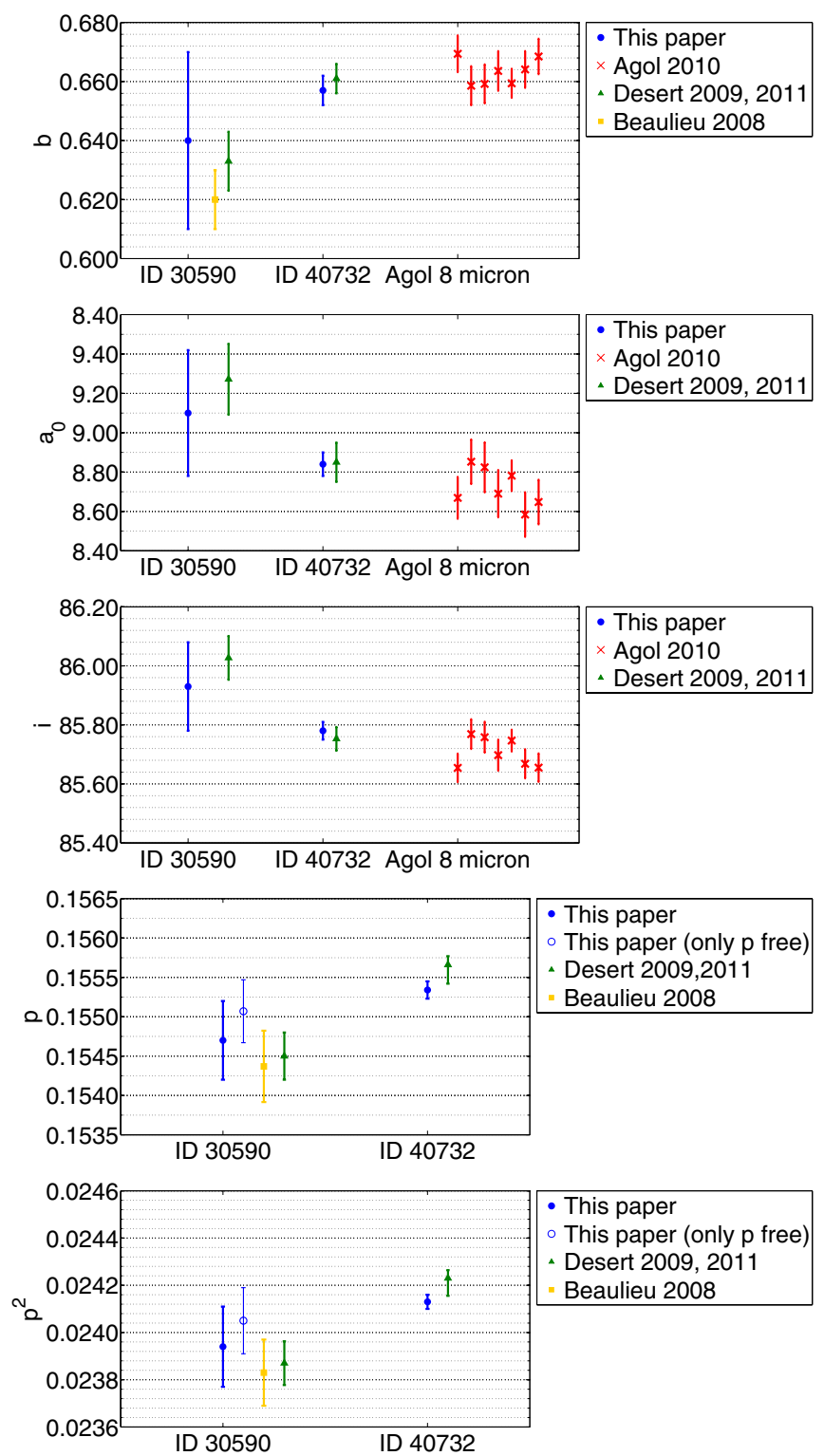

Figure 11. From top to bottom: comparisons of the parameters $b, a_{0}, i, p, p^{2}$, obtained in this paper and in the others discussed here.

(A color version of this figure is available in the online journal.)
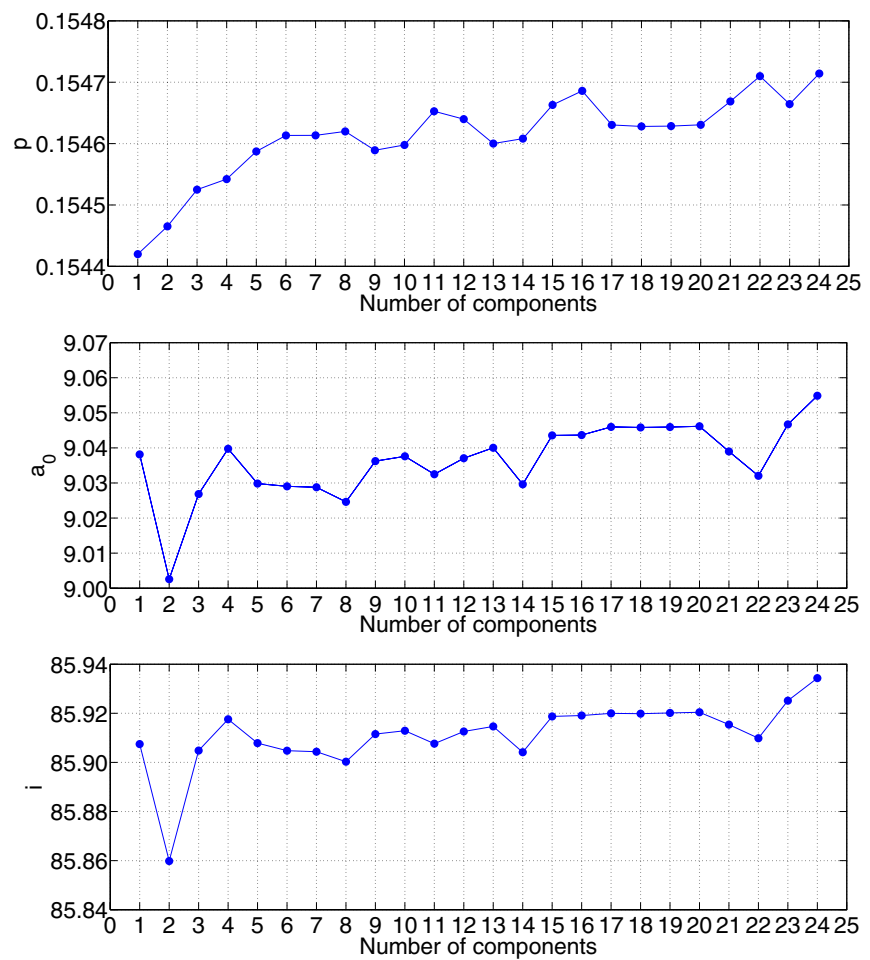

Figure 12. From top to bottom: best values of the parameters $p, a_{0}$, and $i$, respectively, for the transit signals extracted removing the $n$ most significant components from the integral $5 \times 5$ light curve, binned by nine points (ObsID 30590).

(A color version of this figure is available in the online journal.)

\subsection{Comparison with Observations at $8 \mu \mathrm{m}$}

Agol et al. (2010) report a detailed study of seven primary transits and seven secondary eclipses of HD 189733b, observed with Spitzer/IRAC at $8 \mu \mathrm{m}$ (channel 4 of IRAC). Their measured orbital parameters differ from ours by less than the joint $1 \sigma$ uncertainties. Figure 11 includes a comparison of the parameters $a_{0}, i$, and $b$, obtained in this paper with their values. Given the number of primary transits and secondary eclipses they analyzed, and the small impact of the limb darkening effect at $8 \mu \mathrm{m}$, this is a robust confirmation of the validity of our results at $3.6 \mu \mathrm{m}$. We suggest the use of these parameters for future observations at other wavelengths.

Agol et al. (2010) found variations in the transit depth with a range of $\sim 2 \times 10^{-4}$ on $p^{2}$. We could not detect such a difference between the two observations analyzed at $3.6 \mu \mathrm{m}$, as it is comparable with the first error bar.

\subsection{Comparison with Previous Analyses of the Same Observations}

Our results are consistent, at the $1 \sigma$ level, with those of Beaulieu et al. (2008) and Désert et al. (2009) for ID 30590, and Désert et al. (2011) for ID 40732. However, our results afford a substantial agreement (within $1 \sigma$ ) between the transit parameters determined from the two observations, while previous analyses by Désert et al. (2009) and Désert et al. (2011) claimed significant variations of all parameters (e.g., discrepancy $>4 \sigma$ for transit depths). Désert et al. (2011) suggested stellar activity as a possible explanation for those differences. Our results do not support such conclusions, and we find that any possible stellar activity variations are within the error 
Table 5

Best Values of $p, a_{0}$, and $i$ for the Transit Signals Extracted from Different Arrays of Pixels,

Through Method 2, Considering All the Independent Components, Binned by Nine Points

\begin{tabular}{lccccc}
\hline \hline & $3 \times 3$ & $5 \times 5$ & $7 \times 7$ & $9 \times 9$ & $11 \times 11$ \\
\hline$p$ & 0.1549 & 0.1547 & 0.1546 & 0.1547 & 0.1547 \\
$a_{0}$ & 9.02 & 9.05 & 9.07 & 9.06 & 9.07 \\
$i$ & 85.90 & 85.93 & 85.95 & 65.94 & 85.95 \\
\hline$\sigma_{0}$ & $7.1 \times 10^{-4}$ & $6.5 \times 10^{-4}$ & $6.5 \times 10^{-4}$ & $6.5 \times 10^{-4}$ & $6.5 \times 10^{-4}$ \\
$\sigma_{\text {ICA }}$ & $7.8 \times 10^{-4}$ & $7.6 \times 10^{-4}$ & $7.4 \times 10^{-4}$ & $8.2 \times 10^{-4}$ & $8.3 \times 10^{-4}$ \\
\hline$\sigma_{p}$ & 0.00058 & 0.00055 & 0.00054 & 0.00057 & 0.00058 \\
$\sigma_{a_{0}}$ & 0.17 & 0.16 & 0.16 & 0.17 & 0.17 \\
$\sigma_{i}$ & 0.15 & 0.15 & 0.14 & 0.15 & 0.15 \\
\hline
\end{tabular}

Notes. Correspondents $\sigma_{0}$ (computed from the residuals between the signals and the best models), and $\sigma_{\text {ICA. }}$ Derived total standard deviations of the parameters' distributions (ObsID 30590).

Table 6

Best Values of $p, a_{0}$, and $i$ for the Transit Signals Extracted from Different Arrays of Pixels, Through Method 2, Considering All the Independent Components, Binned by Nine Points

\begin{tabular}{lccccc}
\hline \hline & $3 \times 3$ & $5 \times 5$ & $7 \times 7$ & $9 \times 9$ & $11 \times 11$ \\
\hline$p$ & 0.15546 & 0.15534 & 0.15533 & 0.15533 & 0.15528 \\
$a_{0}$ & 8.93 & 8.92 & 8.93 & 8.93 & 8.94 \\
$i$ & 85.79 & 85.78 & 85.79 & 85.79 & 85.79 \\
\hline$\sigma_{0}$ & $1.62 \times 10^{-4}$ & $1.46 \times 10^{-4}$ & $1.45 \times 10^{-4}$ & $1.45 \times 10^{-4}$ & $1.41 \times 10^{-4}$ \\
$\sigma_{\text {ICA }}$ & $1.70 \times 10^{-4}$ & $1.45 \times 10^{-4}$ & $1.60 \times 10^{-4}$ & $1.53 \times 10^{-4}$ & $1.65 \times 10^{-4}$ \\
\hline$\sigma_{p}$ & 0.00013 & 0.00011 & 0.00012 & 0.00011 & 0.00012 \\
$\sigma_{a_{0}}$ & 0.03 & 0.03 & 0.03 & 0.03 & 0.03 \\
$\sigma_{i}$ & 0.03 & 0.03 & 0.03 & 0.03 & 0.03 \\
\hline
\end{tabular}

Notes. Correspondents $\sigma_{0}$ (computed from the residuals between the signals and the best models), and $\sigma_{\text {ICA }}$. Derived total standard deviations of the parameters' distributions (ObsID 40732).
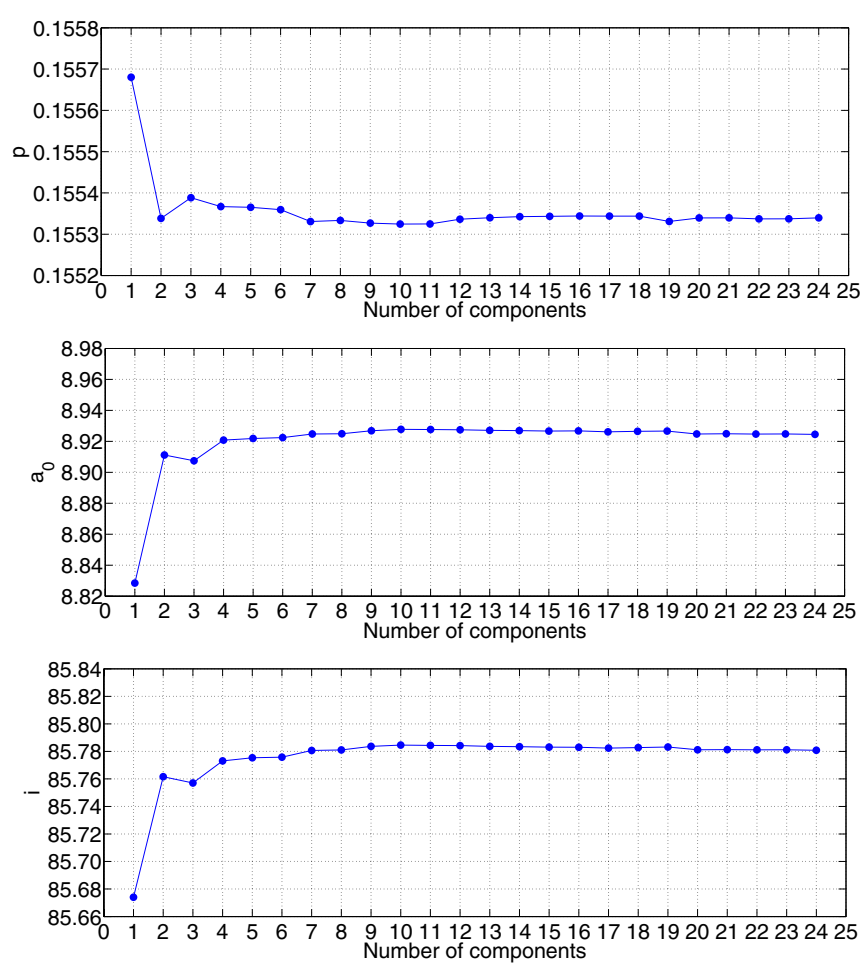

Figure 13. From top to bottom: best values of the parameters $p, a_{0}$, and $i$, respectively, for the transit signals extracted removing the $n$ most significant components, from the integral $5 \times 5$ light curve, binned by nine points (ObsID 40732).

(A color version of this figure is available in the online journal.)

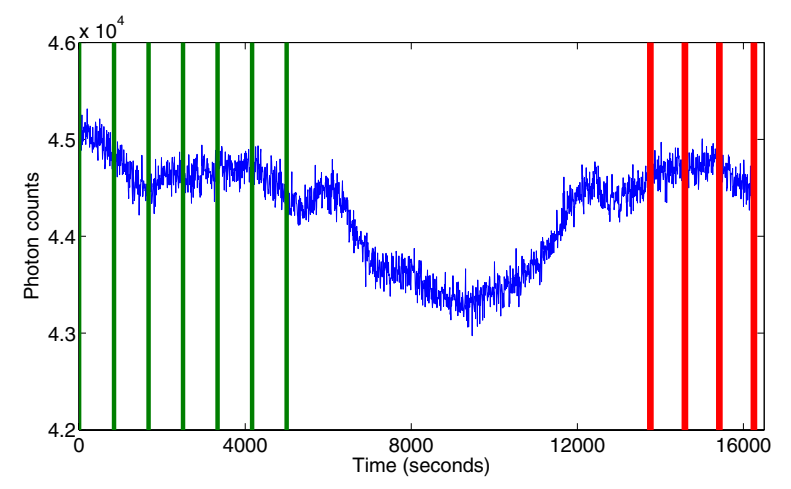

Figure 14. Integral light curve from the $5 \times 5$ array. The green vertical lines indicate the different start points considered; the red vertical lines indicate the end points (ObsID 30590).

(A color version of this figure is available in the online journal.)

bars. Our error bars from ObsID 40732 are of the same order as (for transit depth) or even smaller (for orbital parameters) than in Désert et al. (2011), while those from ObsID 30590 are larger by a factor $\sim 1.6$ with respect to the error bars in Désert et al. (2009). The factor $\sim 1.6$ comes from adding the ICA errors to the parameter error bars derived from the extracted signals. Désert et al. $(2009,2011)$ applied parametric corrections to detrend the transit signals from other disturbances, without attributing any uncertainties to the detrending processes. The fact that we obtained smaller error bars from ObsID 40732, even including the contributions from the detrending process, indicates that, in that context, our blind extraction performed better than their 

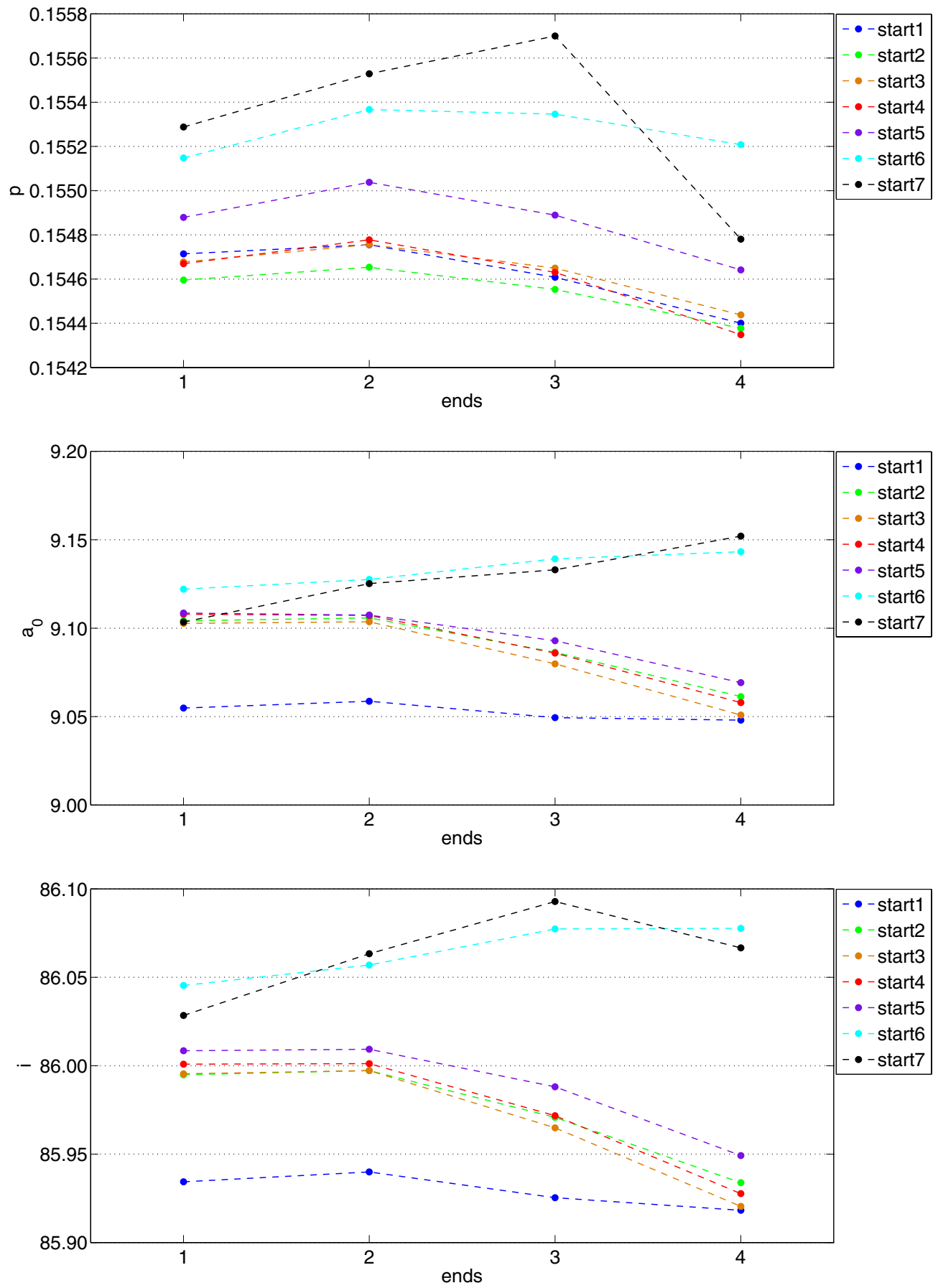

Figure 15. From top to bottom: best values of the parameters $p, a_{0}$, and $i$, respectively, for the transit signals obtained through method 2 , from different sub-data sets. They are extracted using the $5 \times 5$ array, by removing all the independent components from the integral light curve. The curves were binned by nine points before performing the fits. Different colors are used depending on the starts, indexed from earlier to later with increasing integers: blue, start 1; green, start 2; ecru, start 3; red, start 4; purple, start 5; cyan, start 6; black, start 7. Index from 1 to 4 on the horizontal axis indicate different ends, from later to earlier (ObsID 30590).

(A color version of this figure is available in the online journal.)

parameterization. Orbital parameters determined by Beaulieu et al. (2008) and Désert et al. (2009) for ObsID 30590 are not consistent with those for ObsID 40732 obtained by Désert et al. (2011), the results presented here, or the $8 \mu \mathrm{m}$ observations by Agol et al. (2010). Given that the second measurement was superior in quality, and given the agreement with observations at another wavelength, we conclude that the parameters presented in this paper are more robust than those reported by
Beaulieu et al. (2008), or by Désert et al. (2009) using the same data.

We note that Beaulieu et al. (2008) used the same impact parameter at $3.6 \mu \mathrm{m}$ and $5.8 \mu \mathrm{m}$, while Désert et al. (2009) used similar, but not identical, values. Given the conclusions obtained in this paper on the orbital parameters, we suggest that the transit depth at $5.8 \mu \mathrm{m}$ be recalculated accordingly. A reanalysis of the observation at $5.8 \mu \mathrm{m}$ and then of the differences between the transit depths at the two wavelengths, which were 
Table 7

Best Values and Error Bars of $p, a_{0}$, and $i$, Overall Scatters Observed by Using Different Sub-data sets, and by Rejecting the Two Shortest Ones (ObsID 30590)

\begin{tabular}{lccr}
\hline \hline Parameters & Estimated Values & Overall Scatters by Sub-data sets & With Rejections \\
\hline$p$ & $0.1547 \pm 0.0005$ & $0.1543 \div 0.1557$ & $0.1543 \div 0.1550$ \\
$a_{0}$ & $9.05 \pm 0.16$ & $9.05 \div 9.15$ & $9.05 \div 9.11$ \\
$i$ & $85.93 \pm 0.15$ & $85.92 \div 86.09$ & $85.92 \div 86.01$ \\
\hline
\end{tabular}

used to infer about the atmosphere of the planet, should not be strongly affected by this bias, at least in the first case. However, because their conclusions were controversial, a reanalysis of the observation at $5.8 \mu \mathrm{m}$, with more precise orbital parameters and possibly non-parametric technique, as done here, is needed.

\section{CONCLUSIONS}

We have introduced a blind signal-source separation method, based on ICA, to analyze photometric data of transiting exoplanets with a high degree of objectivity; a novel aspect is the use of pixel-light curves, rather than multiple observations.

We have applied the method to reanalyze two Spitzer/IRAC data sets at $3.6 \mu \mathrm{m}$, which previous analyses found gave discrepant results, and obtained consistent transit parameters from the two observations.

We suggest that the large scatter of results reported in the literature arises from

1. use of arbitrary parametric methods to detrend the transit signals, neglecting the relevant uncertainties, and

2. correlations between parameters in the light curve fit.

We found, for ObsID 40732, values for the orbital parameters that are in excellent agreement with those found by Agol et al. (2010), based on Spitzer/IRAC observations at $8 \mu \mathrm{m}$. By applying these values to ObsID 30590, we improved the accuracy of the inferred transit depth, and strengthened the consistency between the two observations.

We thank the anonymous referee for their helpful suggestions G. Morello was partly funded by Erasmus (LLP), "Borse di Studio finalizzate alla ricerca e Assegni finanziati da Programmi Comunitari, decreto $3505 / 2012$ " of Università degli Studi di Palermo, Perren/Impact (CJ4M/CJ0T). G.T. is a Royal Society URF. Part of this work was supported by STFC and ASI-INAF agreement I/022/12/0.

\section{APPENDIX A \\ PARTIAL RESULTS}

\section{A.1. ObsID 30590}

Table 5 reports the best values of the parameters for the transit signals extracted from different arrays of pixels, the standard deviations of the residuals between the signals and the best transit models, and the standard deviations attributed to the ICA separation.

Figure 12 shows the best values of the parameters $p, a_{0}$, and $i$, respectively, for the transit signals extracted removing the $n$ most significant components from the integral $5 \times 5$ light curve, binned by nine points.

\section{A.2. ObsID 40732}

Table 6 reports the best values of the parameters for the transit signals extracted from different arrays of pixels, the standard

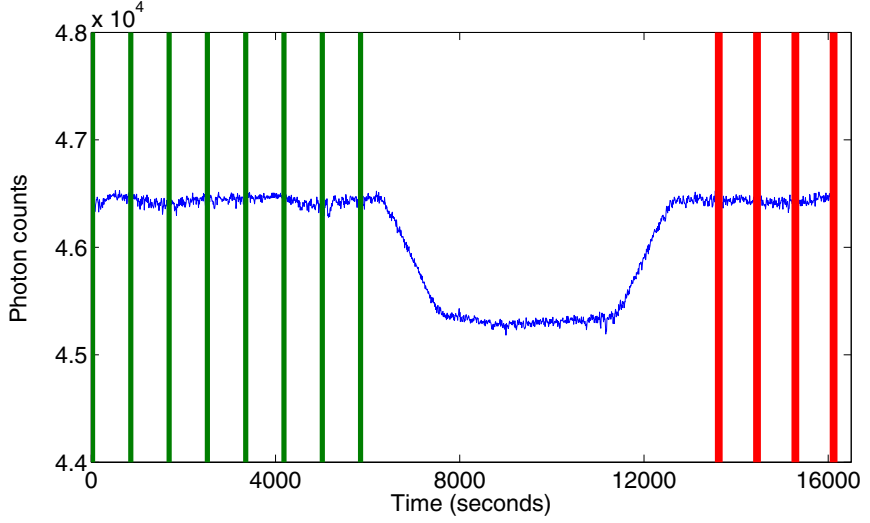

Figure 16. Integral light curve from the $5 \times 5$ array. The green vertical lines indicate the different start points considered; the red vertical lines indicate the end points (ObsID 40732).

(A color version of this figure is available in the online journal.)

deviations of the residuals between the signals and the best transit models, and the standard deviations attributed to the ICA separation.

Figure 13 reports the best values of the parameters $p, a_{0}$, and $i$, respectively, for the transit signals extracted by removing the $n$ most significant components from the integral $5 \times 5$ light curve, binned by nine points.

\section{APPENDIX B}

\section{SUB-DATA SETS}

An important test to verify the robustness of the analyses is to apply the same techniques to sub-data sets. They clearly share the same phenomena, but recorded for different, largely overlapping, time intervals. If the technique is able to separate the source components, the detrended transit signals from different sub-data sets should be essentially equivalent, otherwise there is a problem with at least one of them. A critical factor could be the time length of a sub-data set compared to the timescales of the source signals; for this reason, the separation performed using longer sub-data sets or the whole data set might be more reliable, unless they strengthen some trends or introduce bad data, for example, if they are not well calibrated, or affected by spurious events.

\section{B.1. ObsID 30590}

We considered 28 sub-data sets, obtained by combining seven different starting and four ending times, disposed with regular cadence of $\sim 14$ minutes (see Figure 14).

As before, we used the $5 \times 5$ array, and we applied method 2, by removing all the independent components from the integral light curve. Figure 15 shows the best values of the parameters $p, a_{0}$, and $i$, estimated using each sub-data set.

We can point out some correlations between the best values and both the start and the end points of the sub-data sets. 

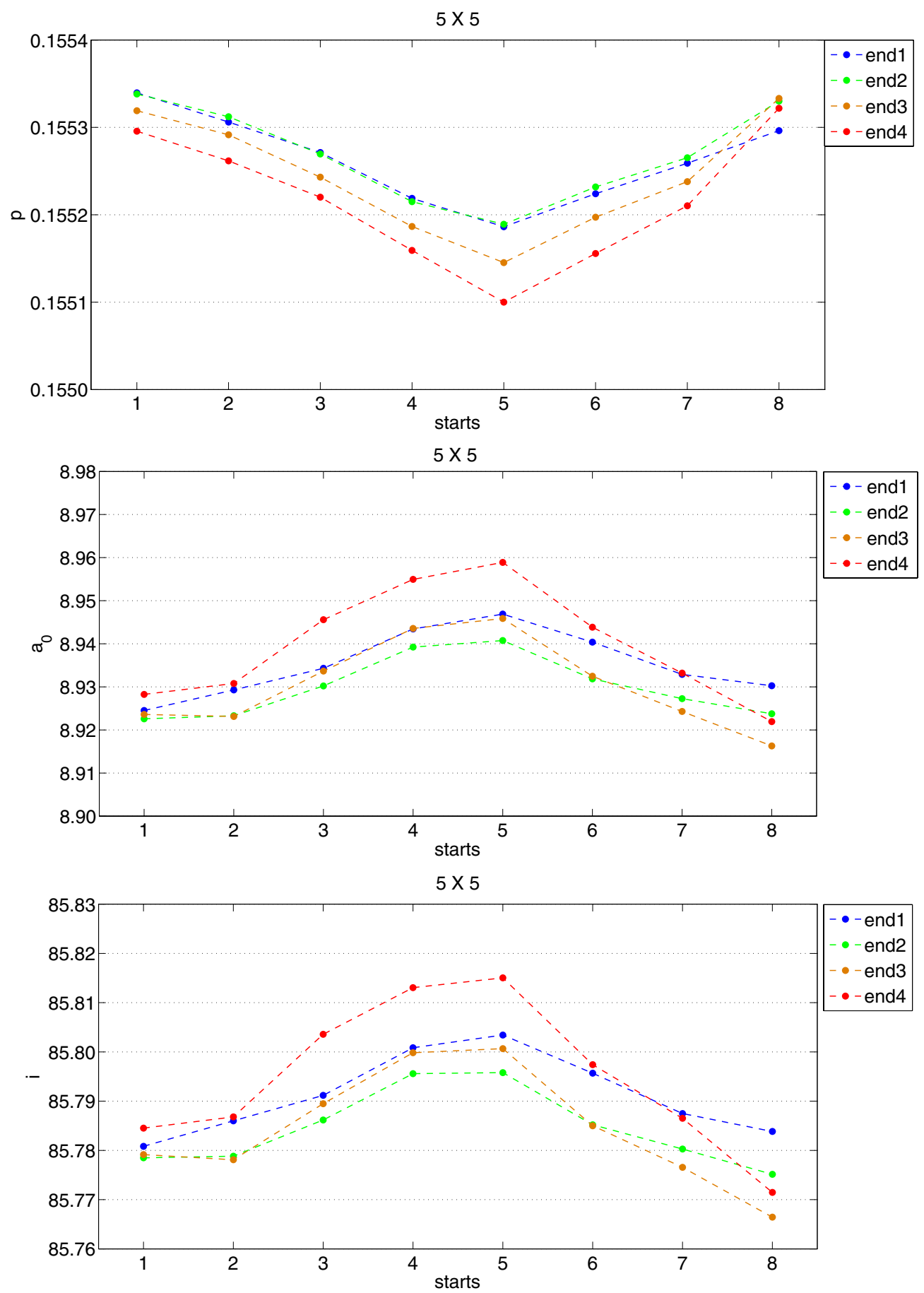

Figure 17. From top to bottom: best values of the parameters $p, a_{0}$, and $i$, respectively, for the transit signals obtained through method 2 , from different sub-data sets. They are extracted using the $5 \times 5$ array, by removing all the independent components from the integral light curve. The curves were binned by nine points before performing the fits. Different colors are used depending on the ends, indexed from later to earlier with increasing integers: blue, end 1; green, end 2; ecru, end 3; red, end 4. Index from 1 to 8 on the horizontal axis indicates different starts, from later to earlier (ObsID 40732).

(A color version of this figure is available in the online journal.)

The overall scatters are compatible with the ranges determined before. Table 7 reports the estimated ranges of the parameters with the scatters observed by the sub-data sets, either by including or by rejecting the two shortest sub-data sets.

\section{B.2. ObsID 40732}

We considered 32 sub-data sets, obtained by combining eight different starting times and four ending times, disposed with regular cadence of $\sim 14$ minutes (see Figure 16).
As usual, we used the $5 \times 5$ array, and we applied method 2 , and removed all the independent components from the integral light curve. Figure 17 shows the best values of the parameters $p, a_{0}$, and $i$, estimated using each sub-data set.

Again, there are some correlations between the best values and the extremes of the sub-data sets, but the overall scatters are compatible with the ranges previously estimated. Table 8 reports the estimated ranges of the parameters with the scatters observed by the sub-data sets. 
Table 8

Best Values and Error Bars of $p, a_{0}$, and $i$, Overall Scatters Observed by Using Different Sub-data sets (ObsID 40732)

\begin{tabular}{lcc}
\hline \hline Parameters & Estimated Values & Overall Scatters by Sub-data sets \\
\hline$p$ & $0.15534 \pm 0.00011$ & $0.15510 \div 0.15534$ \\
$a_{0}$ & $8.92 \pm 0.03$ & $8.92 \div 8.96$ \\
$i$ & $85.78 \pm 0.03$ & $85.77 \div 85.82$ \\
\hline
\end{tabular}

Table 9

Estimated Best Values and Error Bars of $p, a_{0}, i, p^{2}, b$, and $T$ by Applying Method 1 and Method 2 (Both Observations)

\begin{tabular}{lcc}
\hline \hline ID 30590 & Method 1 & Method 2 \\
\hline$p$ & $0.1547 \pm 0.0019$ & $0.1547 \pm 0.0005$ \\
$a_{0}$ & $9.1 \pm 0.5$ & $9.05 \pm 0.16$ \\
$i$ & $85.9 \pm 0.5$ & $85.93 \pm 0.15$ \\
$p^{2}$ & $0.0239 \pm 0.0006$ & $0.02394 \pm 0.00017$ \\
$b$ & $0.64 \pm 0.11$ & $0.64 \pm 0.03$ \\
$T$ & $5160 \pm 900 \mathrm{~s}$ & $5170 \pm 200 \mathrm{~s}$ \\
\hline ID 40732 & Method 1 & Method 2 \\
\hline$p$ & $0.1553 \pm 0.0004$ & $0.15534 \pm 0.00011$ \\
$a_{0}$ & $8.96 \pm 0.10$ & $85.78 \pm 0.03$ \\
$i$ & $85.81 \pm 0.11$ & $0.02413 \pm 0.00003$ \\
$p^{2}$ & $0.02413 \pm 0.00012$ & $0.657 \pm 0.005$ \\
$b$ & $0.654 \pm 0.019$ & $5157 \pm 34 \mathrm{~s}$ \\
$T$ & $5156 \pm 124 \mathrm{~s}$ &
\end{tabular}

\section{APPENDIX C \\ METHOD 1: DIRECT IDENTIFICATION OF THE TRANSIT COMPONENT}

Table 9 reports the results obtained by applying method 1 and method 2 on both observations, using the whole data sets, and the $5 \times 5$ arrays. It is straightforward to note that the best values are almost coincident, but the uncertainties derived with method 1 are larger by a factor $\sim 3 \div 4$. The differences are due to the ICA contributions to the error bars.

We also observed that, in these cases, the transit signals estimated with method 2 tend toward the ones obtained by method 1 , when increasing the number of non-transit-components removed; this is shown in Figure 18.

However, the larger error bars provided by the ICA terms are justified by the scatters obtained using different arrays of pixels and different sub-data sets. We do not report the results in detail, but we summarize the main facts observed.

1. In some cases, the transit component is clearly corrupted, discouraging quantitative analysis.

2. The scatters of the transit parameters obtained by using different sub-data sets are comparable with the error bars estimated (the arrays of pixels play a minor role, but more important than if using method 2).

3. For longer sub-data sets, which are expected to allow better extractions of the independent components, the results obtained with methods 1 and 2 tend to agree.
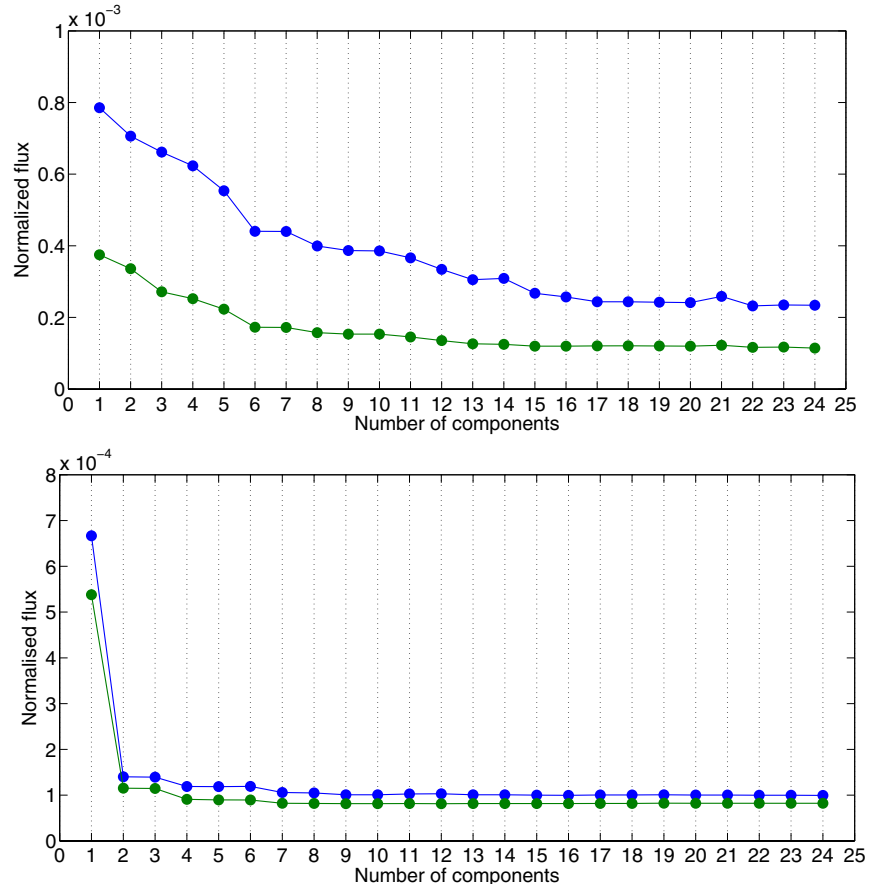

Figure 18. Top: ObsID 30590; blue, mean quadratic deviations between the transit signals estimated through method 2, with the $n$ most important components, and the one estimated through method 1 , using the $5 \times 5$ array; green, the same, considering the binned signals. Bottom: the same for ObsID 40732.

(A color version of this figure is available in the online journal.)

\section{REFERENCES}

Agol, E., Cowan, N. B., Knutson, H. A., et al. 2010, ApJ, 721, 1861 Ballerini, P., Micela, G., Lanza, A. F., \& Pagano, I. 2012, A\&A, 539, A140 Beaulieu, J. P., Carey, S., Ribas, I., \& Tinetti, G. 2008, ApJ, 677, 1343 Berta, Z. K., Charbonneau, D., Bean, J., et al. 2011, ApJ, 736, 12 Brown, T. M. 2001, ApJ, 553, 1006

Collier Cameron, A., Bruce, V. A., Miller, G. R. M., Triaud, A. H. M. J., \& Queloz, D. 2010, MNRAS, 403, 151

Désert, J. M., Lecavelier des Etangs, A., Hébrard, G., et al. 2009, ApJ, 699, 478

Désert, J. M., Sing, D. K., Vidal-Madjar, A., et al. 2011, A\&A, 526, A12

Ehrenreich, D., Guillaume, H., Lecavelier des Etangs, A., et al. 2007, ApJ, 668,179

Haario, H., Laine, M., Mira, A., \& Saksman, E. 2006, Stat. Comput., 16, 339

Howarth, I. D. 2011, MNRAS, 418, 1165

Hyvärinen, A., Karhunen, J., \& Oja, E. 2001, Independent Component Analysis (New York: Wiley)

Kipping, D. M. 2008, MNRAS, 389, 1383

Koldovský, Z., Tichavský, P., \& Oja, E. 2006, ITNN, 17, 1265

Lagarias, J. C., Reeds, J. A., Wright, M. H., \& Wright, P. E. 1998, SIAM J. Optim., 9, 112

Mandel, K., \& Agol, E. 2002, ApJL, 580, L171

Seager, S., \& Mallén-Ornelas, G. 2003, ApJ, 585, 1038

Seager, S., \& Sasselov, D. D. 2000, ApJ, 537, 916

Tichavský, P., Koldovský, Z., Yeredor, A., Gómez-Herrero, G., \& Doron, E. 2008, ITNN, 19, 421

Tinetti, G., Liang, M., Vidal-Madjar, A., et al. 2007a, ApJL, 654, L99

Tinetti, G., Vidal-Madjar, A., Liang, M. C., et al. 2007b, Natur, 448, 169

Triaud, A. H. M. J., Collier Cameron, A., Queloz, D., et al. 2010, A\&A, 524, A25

Waldmann, I. P. 2012, ApJ, 747, 12

Waldmann, I. P., Tinetti, G., Deroo, P., et al. 2013, ApJ, 766, 7

Yeredor, A. 2000, ISPL, 7, 197 